\title{
RESULTADOS DEL TRATAMIENTO DE LA PROLIFERACIÓN ANGIOMATOSA RETINIANA MEDIANTE TERAPIA FOTODINÁMICA
}

\section{RESULTS OF TREATMENT OF RETINAL ANGIOMATOUS PROLIFERATION WITH PHOTODYNAMIC THERAPY}

\author{
SALAZAR-DÍEZ JL ${ }^{1}$, ITURRALDE-ERREA D ${ }^{1}$, DÍAZ-DE-DURANA-SANTACOLOMA E ${ }^{1}$, \\ FERNÁNDEZ-ARES ML ${ }^{1}$, VÁZQUEZ-CRUCHAGA E ${ }^{1}$, LÓPEZ-GARRIDO JA ${ }^{1}$
}

\begin{abstract}
RESUMEN
Propósito: Determinar el efecto de la terapia fotodinámica con el uso de verteporfino en el tratamiento de pacientes con proliferación angiomatosa retiniana (PAR) y la incidencia de esta patología retiniana en nuestro medio.

Métodos: Se presenta un estudio retrospectivo de 11 casos diagnosticados de PAR y que fueron tratados con terapia fotodinámica con verteporfino.

Resultados: La incidencia de PAR en el grupo de ojos con neovascularización coroidea subfoveal mínimamente clásica u oculta fue del $8 \%$. El seguimiento medio fue de 15 meses. La AV mejoró en 3, permaneció igual en 4 , y disminuyó en 4 . 4 pacientes presentaban una lesión bilateral.

Conclusiones: La proliferación angiomatosa retiniana no tiene un tratamiento demostrado. Estos resultados sugieren que TFD puede ser una terapia útil en pacientes con PAR y parece reducir el riesgo de pérdida visual.
\end{abstract}

Palabras clave: Macula, degeneración macular asociada a la edad, proliferación angiomatosa retiniana, terapia fotodinámica, neovascularización coroidea.

\begin{abstract}
Purpose: To determine the effects of phothodynamic therapy using verteporfin in the treatment of patients with retinal angiomatous proliferation (RAP) and the incidence of this retinal disease in our area.

Methods: We performed a retrospective study of 11 cases of RAP who were treated with photodynamic therapy using verteporfin (PDT).

Results: The incidence of RAP in the group of eyes with minimally classic or occult subfoveal choroidal neovascularization was $8 \%$. The mean followup time after treatment was 15 months. The visual acuity improved in 3 , remained the same in 4 , and decreased in 4. In 4 patients, angiomatous lesions were observed in both eyes.

Conclusions: There is no method proven to be effective for the treatment of retinal angiomatous proliferation. Our results suggest that PDT may be useful therapy in patients with RAP as it appeared to reduce the risk of visual loss (Arch Soc Esp Oftalmol 2006; 81: 401-404).
\end{abstract}

Key words: Macula, age-related macular degeneration, retinal angiomatous proliferation, photodynamic therapy, choroidal neovascularization.

Recibido: 10/11/05. Aceptado: 19/7/06.

Servicio de Oftalmología. Hospital de Galdácano. Vizcaya. España.

1 Licenciado en Medicina.

Comunicación presentada en el LXXXI Congreso de la S.E.O. (Zaragoza 2005).

Correspondencia:

José Luis Salazar Díez

Hospital de Galdakao

Barrio Labeaga, s/n

48960 Galdakao (Vizcaya)

España

E-mail: jlsalazar@ono.com 


\section{INTRODUCCIÓN}

La proliferación angiomatosa retiniana (PAR, RAP en la literatura anglosajona) se puede definir como una forma distinta de degeneración macular asociada a la edad (DMAE) neovascular que se asocia con proliferación de capilares retinianos y en la que se identifican varios estadios evolutivos. En estos casos la proliferación capilar retiniana puede progresar más allá de los límites de la retina para dar lugar a neovascularización subretiniana (1-3).

El comportamiento angiográfico de la PAR es el de una membrana neovascular de subtipo mínimamente clásico u oculta, pudiendo superponerse a un desprendimiento del epitelio pigmentario (fig. 1). En la angiografía con verde de indocianina (AVI) se identifica como un hot spot (fig. 2).

En la presente publicación se valora la incidencia de la PAR en nuestro medio así como nuestros resultados en el tratamiento mediante TFD.

\section{SUJETOS, MATERIAL Y MÉTODOS}

Se plantea un estudio retrospectivo sobre un total de 537 ojos consecutivos tratados en este Servicio mediante terapia fotodinámica (TFD) desde febrero de 2003 a febrero de 2005. Los pacientes eran todos mayores de 55 años y presentaban alteraciones de fondo de ojo compatibles con DMAE.

Cada uno de los ojos identificados como PAR recibió tratamiento mediante TFD con verteporfino

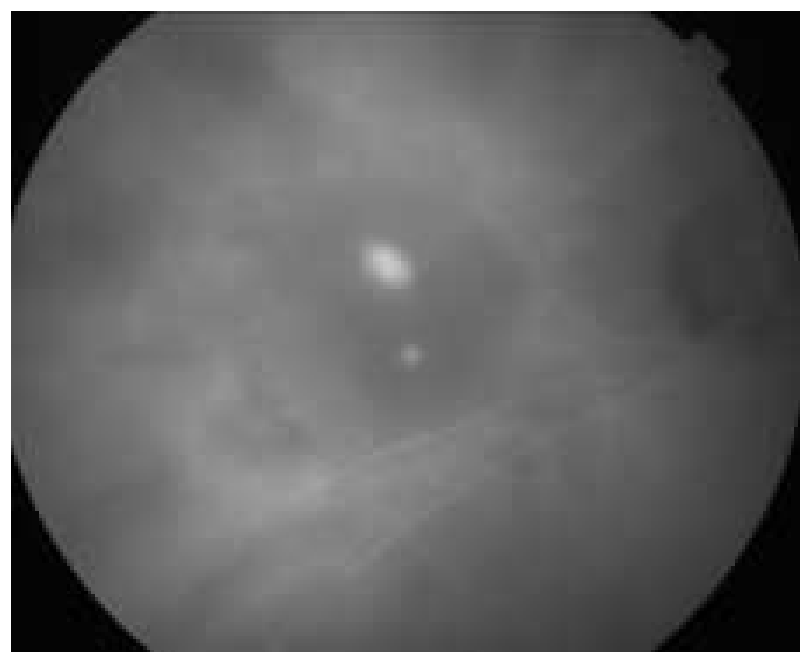

Fig. 1: Angiografía con verde de indocianina (AVI) previa a tratamiento en uno de los casos.

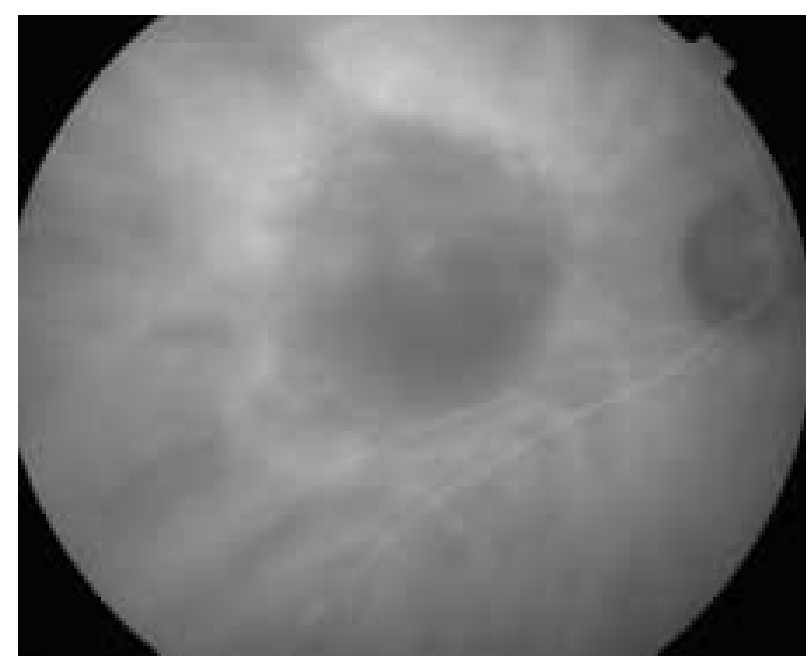

Fig. 2: AVI tras el tratamiento del mismo caso de la figura 1 .

tras la correcta identificación de la lesión mediante angiografía fluoresceínica en todos los casos, y con verde de indocianina cuando se consideró necesaria para una adecuada valoración. Las administraciones posteriores de TFD se realizaron con un intervalo mínimo de 3 meses tras evaluar la presencia de escape mediante biomicroscopía de fondo de ojo y angiografía fluoresceínica con o sin verde de indocianina asociada. En todos los pacientes se obtuvo consentimiento informado para la realización de angiofluoresceingrafía (AGF), AVI y TFD.

La preparación y administración del verteporfino se hizo de forma idéntica a la usada en el ensayo clínico TAP. En un caso se realizó un ajuste del spot en función de la AVI en un intento de evitar posibles complicaciones en un desprendimiento de epitelio pigmentario de gran tamaño (fig. 3). La cuantificación de la agudeza visual (AV) previa y postratamiento se realizó mediante optotipos de Snellen y los datos fueron analizados de forma retrospectiva.

En todos los casos se realizó una angiografía fluoresceínica y en 4 pacientes una AVI para su diagnóstico.

\section{RESULTADOS}

Se identificaron un total de 12 ojos correspondientes a 9 pacientes con presencia de una PAR sobre un total de 537 ojos tratados (fig. 4). De estos 


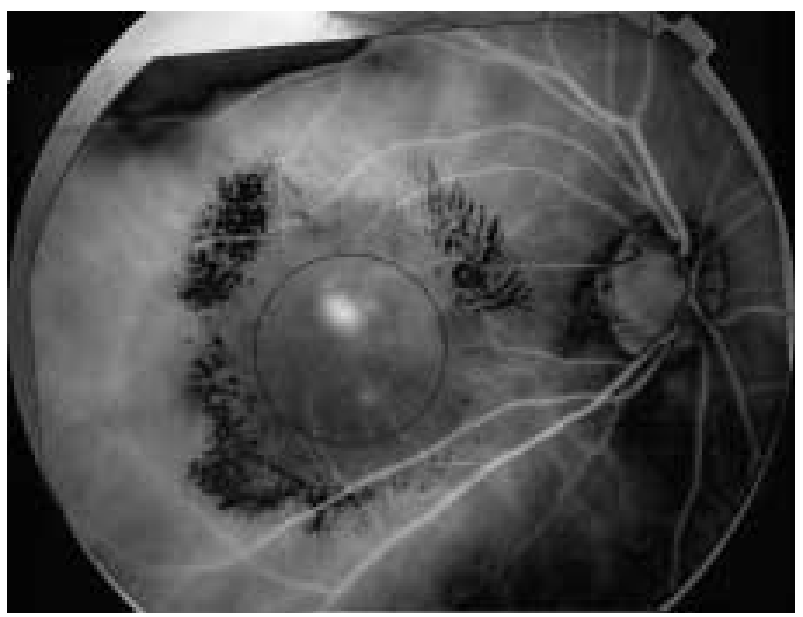

Fig. 3: Fotomontaje del caso de la figura 1 de AVI y aneritra para guía del tratamiento.

537 ojos 148 correspondían a membranas ocultas o mínimamente clásicas. La media de edad de los 9 pacientes fue de 83 años. 7 pacientes eran mujeres (77\%) y 2 hombres (23\%). En los 4 pacientes bilaterales la neovascularización desarrollada fue una PAR en ambos ojos y 3 de ellos fueron tratados bilateralmente. Un paciente (el último identificado) presentó un seguimiento menor de 1 mes y en él se había realizado un tratamiento mediante triancinolona intravítrea asociada a terapia fotodinámica por lo que no se le consideró para la valoración de resultados. Los 11 ojos restantes tenían un seguimiento medio de 15 meses con un rango de 9 meses a 24 meses. 4 de los 11 ojos presentaron una AV

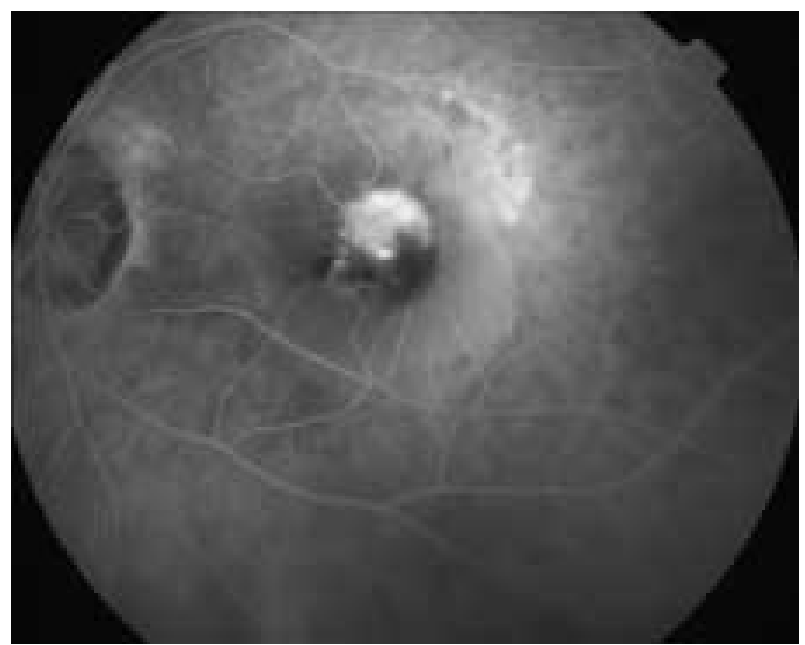

Fig. 4: Angiografía fluoresceínica del otro ojo, más evolucionado, del mismo paciente. igual al final del seguimiento, 3 mejoraron 1 línea o más en su AV y 4 empeoraron 1 línea o más. 6 ojos seguían en tratamiento en el momento de la valoración de resultados. De los 5 ojos con tratamiento que se consideró finalizado, 2 presentan peor $\mathrm{AV}, 2$ la misma $\mathrm{AV}$ y 1 caso mejoró.

\section{DISCUSIÓN}

En el presente estudio valoramos nuestra experiencia clínica en el tratamiento de la proliferación angiomatosa retiniana mediante terapia fotodinámica.

Hemos identificado un total de 12 ojos que presentan una proliferación angiomatosa retiniana sobre un total de 537 ojos tratados mediante terapia fotodinámica lo que supone un $2 \%$ del total. Hay que aclarar que en todo momento se han seguido las guías de tratamiento definidas por los estudios TAP y VIP con lo que los pacientes que presentaron una membrana oculta o mínimamente clásica fueron una indicación de tratamiento más reciente. Si nos referimos a aquellos ojos que se incluían en uno de estos dos últimos subgrupos la incidencia de PAR supone un $8 \%$ de este subtotal.

Nuestro centro es uno de los dos de referencia existentes en nuestra comunidad para el tratamiento mediante TFD, proporcionando la cobertura sanitaria en el tratamiento de la DMAE a una población aproximada de 1.400.000 habitantes. Es posible por ello que la incidencia se encuentre subestimada en función de diferentes criterios a la hora de la remisión de pacientes.

La existencia de un mayor número de mujeres entre los pacientes afectos (77\%) y la edad avanzada (media de 83 años) ya ha sido señalada por otros autores (3). Es probable que este proceso sea más edad dependiente que la DMAE en general y que la mayor esperanza de vida que presentan las mujeres esté determinando un sesgo respecto al sexo.

Llama la atención que los casos bilaterales (fig. 4) presentaron el mismo tipo de lesión (proliferación angiomatosa retiniana) en ambos ojos indicando con ello que estamos probablemente ante un proceso con características diferenciales respecto a otras alteraciones neovasculares asociadas a la edad (4).

Se han planteado múltiples alternativas terapéuticas en el tratamiento de la proliferación angiomatosa retiniana. Los estadios iniciales son en general poco sintomáticos presentándose muchas veces 
como lesiones complicadas con un proceso neovascular coroideo.

Se ha realizado fotocoagulación mediante láser verde guiada por angiografía con verde de indocianina de alta velocidad (comunicación Murphy R en el subspecialty day-AAO 2005 en Chicago). Se puede argumentar en contra que la existencia de un flujo dependiente de las dos circulaciones puede dificultar el cierre vascular. A ello puede colaborar la existencia de un estimulo angiogénico mayor en el medio por la propia naturaleza de la patología.

La cirugía submacular se vería en principio dificultada por la presencia de conexiones entre coroides y retina que pudieran llevar a roturas yatrógenas. Se han evidenciado mejorías en casos puntuales mediante la realización de corte selectivo de la vénula y arteriola de alimentación con microtijeras de vitrectomía (5).

Se ha comunicado también el efecto del tratamiento mediante terapia fotodinámica en PAR asociada a desprendimiento del epitelio pigmentario (6), debiendo tener especial cautela en aquellos casos en que un desprendimiento del epitelio pigmentario sea el componente mayoritario de la lesión por la posibilidad de rotura.

En nuestro estudio un $64 \%$ de los pacientes presentan una AV estable, considerada esta como perdida menor de una línea o mejoría, con 15 meses de seguimiento medio. Estos resultados se comparan favorablemente con los resultados del estudio VIP en membranas ocultas que refieren una situación entendida por ellos como estable (pérdida menor de 3 líneas de ETDRS) de 49,3\% de los casos (7).

Concluimos por tanto que en nuestra experiencia clínica los resultados del tratamiento mediante tera- pia fotodinámica de la proliferación angiomatosa retiniana son buenos a corto-medio plazo, estando por determinar si ese efecto se mantiene en el tiempo y siendo conscientes de las limitaciones inherentes a un estudio retrospectivo con tan escaso número de casos.

\section{BIBLIOGRAFÍA}

1. Hartnett ME, Weiter JJ, Garsd A, Jalkh AE. Classification of retinal pigment epithelial detachments associated with drusen. Graefes Arch Clin Exp Ophthalmol 1992; 230: 11-19.

2. Hartnett ME, Weiter JJ, Staurenghi G, Elsner AE. Deep retinal vascular anomalous complexes in advanced agerelated macular degeneration. Ophthalmology 1996; 103: 2042-2053.

3. Yannuzzi LA, Negrao S, Iida T, Carvalho C, RodriguezColeman H, Slakter J, et al. Retinal angiomatous proliferation in age-related macular degeneration. Retina 2001; 21: 416-434.

4. Gross N, Aizman A, Brucker A, Klancnik J Jr, Yannuzzi LA. Nature and risk of neovascularization in the fellow eye of patients with unilateral retinal angiomatous proliferation. Retina 2005; 25: 713-718.

5. Borrillo JL, Sivalingam A, Martidis A, Federman JL. Surgical ablation of retinal angiomatous proliferation. Arch Ophthalmol 2003; 121: 558-561.

6. Boscia F, Furino C, Sborgia L, Reibaldi M, Sborgia C. Photodynamic therapy for retinal angiomatous proliferations and pigment epithelium detachment. Am J Ophthalmol 2004; 138: 1077-1079.

7. Verteporfin In Photodynamic Therapy Study Group. Verteporfin therapy of subfoveal choroidal neovascularization in age-related macular degeneration: two-year results of a randomized clinical trial including lesions with occult with no classic choroidal neovascularization--verteporfin in photodynamic therapy report 2. Am J Ophthalmol 2001; 131: $541-560$. 